Article

\title{
The Association between Health-Related Fitness and Physical Activity during Weekdays: Do Fit Students Exercise More after School?
}

\author{
Dehong Yang $\left.{ }^{1} \mathbb{(}\right)$, Xihe Zhu ${ }^{2}$, Justin A. Haegele ${ }^{2} \mathbb{C}$, Patrick B. Wilson ${ }^{2} \mathbb{D}$ and Xueping $\mathrm{Wu}^{3, *}$ \\ 1 Department of Physical Education, Shanghai Institute of Technology, Shanghai 201418, China \\ 2 Department of Human Movement Sciences, Old Dominion University, Norfolk, VA 23529, USA \\ 3 School of Physical Education and Sport Training, Shanghai University of Sport, Shanghai 200438, China \\ * Correspondence: wuxueping@sus.edu.cn
}

Received: 5 July 2019; Accepted: 29 July 2019; Published: 31 July 2019

\begin{abstract}
Physically active students are more likely to be fit and learn better in school than sedentary ones. For school-aged children, it is unclear whether physical fitness level is an important determinant for student's activity afterschool. This study examined the association between health-related physical fitness test performance and afterschool physical activity during weekdays. Participants $(n=97$, 11-13 years old) completed health-related fitness tests. They wore Actigraph GT3X accelerometers for five consecutive days, recording physical activity participation during after school hours. Descriptive statistics for both afterschool physical activity and health-related fitness were summarized. Regression analyses were conducted to determine the association between variables. Participants spent most of their afterschool hours participating in sedentary behaviors $(274.27 \pm 66.89 \mathrm{~min})$ and light physical activity $(73.68 \pm 51.66 \mathrm{~min})$, and only $11.35 \pm 16.92 \mathrm{~min}$ of moderate-to-vigorous physical activity (MVPA), accumulating approximately $2058.52 \pm 1690.56$ steps each day afterschool. The regression model explained $22.8 \%$ of the variance in afterschool MVPA, and Progressive Aerobic Cardiovascular Endurance Run (PACER) scores were the only statistically significant predictor $(\beta=0.47, p<0.01)$. Fit students do tend to exercise more after school. Promoting physical fitness in school is an important factor for students' afterschool physical activity participation during weekdays.
\end{abstract}

Keywords: adolescent; exercise promotion; cardiorespiratory endurance; health-related fitness; physical education

\section{Introduction}

Physical activity and health-related fitness have been documented to have many physical and psychological benefits among school-aged children [1,2]. As such, government agencies and professional associations recommend that children should participate in at least $60 \mathrm{~min}$ of moderate-to-vigorous physical activity (MVPA) [3]; and improving students' physical fitness is set as a goal for physical education in the United States [4]. As the instructional time for physical education declines, it is reasonable for researchers and practitioners to look for opportunities after school to promote physical activity, and at the same time examine current fitness promotion (e.g., regular testing) in schools. Despite the decline in physical education instruction time, the United States Government Accountability Office (GAO) report suggests that increased sport programs and other physical activity opportunities during afterschool hours should play an important role in improving students' weekly physical activity [5]. While physical activity opportunities may exist after school, coherent instruction on physical fitness or physical activity may not be available many students. Bridging what students have achieved in physical education (e.g., fitness) to their afterschool behavior will be critical. After all, 
from the perspectives of physical educators, health-related fitness testing is a measure conducted during instruction time, with an ultimate goal of promoting fitness and lifetime voluntary physical activity participation [4].

Health-related fitness tests are recommended as the essential element of fitness education in physical education [6]. In over $60 \%$ of public schools in the United States, health-related fitness testing is a customary element of physical education programs [7]. These field-based health-related fitness tests often include several components [8]: body composition (e.g., body mass index, BMI), cardiorespiratory endurance (e.g., one-mile run or Progressive Aerobic Cardiovascular Endurance Run (PACER) test), flexibility (e.g., sit-and-reach), and muscular strength/endurance (e.g., pushup, curl-up, or trunk-lift). Given that these fitness tests are widely conducted in schools, questions exist regarding the value and contribution of these tests to physical activity promotion. Currently, there is a lack of strong evidence on the role of fitness testing in promoting habitual physical activity participation $[9,10]$.

A number of studies have examined the relationship between daily physical activity participation and physical fitness, particularly body composition as well as cardiorespiratory endurance [11-16]. Among the earlier studies, Morrow and Freedson reviewed research relating adolescent physical activity (mostly self-reported) and cardiorespiratory endurance (either laboratory or field-tested) and they found only low correlation coefficients $(r=0.16-0.17)$ between these variables [12]. They suggested that more physical activity tracking and measurement was needed, and that light physical activity was unlikely to be associated with cardiorespiratory endurance. Since this report, physical activity and health-related fitness measurement techniques have been substantially improved [17]. For instance, accelerometry and other objective portable measures are now readily available for research and everyday usage.

While studies have shown that physical activity, particularly moderate-to-vigorous physical activity (MVPA) tends to positively predict cardiorespiratory fitness [13,14], a recent review reported mixed findings regarding the relation between health-related fitness and physical activity from 14 studies published after 2000 [15]. For example, Aires and colleagues (2010) reported a significant positive correlation between adolescents' vigorous physical activity and PACER performance $(\mathrm{emphr}=0.39$ ), but no significant correlation with light or moderate physical activity [11]. Similarly, Ross and Mcguire found that incidental moderate physical activity (accelerometry-measured) positively predicted cardiorespiratory fitness $\left(r^{2}=0.20\right)$ in adult populations. Further they suggested that health-related fitness could be a mediator between physical activity and obesity [16].

It is known that physical activity intervention can improve physical fitness, particularly cardiorespiratory endurance [18]. It is also known from the correlational studies that habitual physical activity participation positively predicts physical fitness among children and adolescents [15-17]. For students in schools, however, their school time is often tightly scheduled during weekdays that the primary time available for voluntary activities is afterschool hours. Among the existing studies cited $[11,13,14,16]$, all have collected physical activity data from the whole day period either from a self-report or more objective measures. What is unknown is whether students' physical fitness levels predict their afterschool physical activity participation. That is, do fit students tend to exercise more after school? Therefore, this study aims to address whether fit students tend to exercise more afterschool by examining the association between health-related fitness performance and afterschool physical activity participation during weekdays. Specifically, the study seeks to examine whether adolescents' health-related fitness performances predict accelerometer-measured afterschool moderate-to-vigorous physical activity. Identifying the association would help uncover the link, or lack thereof, between these two important variables related to physical education in schools. Likewise, establishing a link between health-related fitness testing and afterschool physical activity would provide further support for the value of fitness testing/education in schools. Furthermore, fitness testing performance in childhood and adolescence is associated with physical activity engagement later in life $[19,20]$, providing additional support for using physical education-based fitness programs as a positive correlate to promote lifelong physical activity participation. 


\section{Materials and Methods}

\subsection{Sample}

The participants of this study were seventh graders $(N=97)$ including 49 females and 48 males. Based on the anticipated effect size $\mathrm{f}^{2}=0.15$, statistical power level of 0.80 with seven predictors and $\alpha=0.05$, the required sample size estimation would be 103. Average participant age was 12.41, $\mathrm{SD}=0.44$ years old, ranging from 11 to 13 . Based on the student self-report, the race/ethnic distribution of the sample was 6.3\% Asian American, 12.1\% African/Black American, 66\% Caucasian/White, 10.6\% Hispanic/Latino, and 5\% other ethnic groups. The sample reflected the diverse general population of the school in Eastern United States. In order to participate in the study, the students had to be free of physical or mental impairments which may prevent them being able to attend physical education independently or complete the physical fitness tests. Participants completed and returned their assent form and their parent/guardian returned consent forms to indicate their willingness to voluntarily participate in the study prior to data collection. Both the university institutional review board and school district research office approved the study protocols.

\subsection{Measures and Procedures}

\subsubsection{Demographic Characteristics}

A self-reported 10-question demographic questionnaire was used to gather participant demographic characteristics. These questions asked the students to provide information pertaining to (a) age, (b) sex, (c) race/ethnicity, (d) grade level, and (e) whether they have disabilities or physical conditions that may prevent them from completing the fitness assessments. Those who indicated having disabilities or physical conditions were not included in the study.

\subsubsection{Health-Related Fitness}

The field-based measures of health-related fitness components were conducted in this study, including: body composition, cardiorespiratory endurance, muscular strength/endurance, and flexibility [8].Specifically, body composition was measured using body mass index through height and weight (i.e., weight/height ${ }^{2}$ ). Height was measured using a portable stadiometer, and weight was measured using a balance beam scale (Dectecto 437, New York, NY, USA). For cardiorespiratory fitness, the 20-meter PACER (i.e., shuttle run) test was conducted, and the number of completed laps was recorded as performance. For muscular strength and endurance, the 90-degree pushup, and curl-up were conducted with cadence from the Cooper Institute [8]. For flexibility, we conducted the trunk-lift and back-saver sit-and-reach test, where the best performance of two trials were recorded. These measures are often tested in the schools and are reported with acceptable reliability and validity [8].

\subsubsection{Afterschool Physical Activity}

ActiGraph $^{\mathrm{TM}}$ tri-axial accelerometers (Model: GT3X, ActiGraph, Pensacola, FL, USA) were used to collect student afterschool physical activity participation. GT3X accelerometers are widely used in physical activity (PA) research, and they are valid and reliable measures for generating activity counts, step counts, and calorie expenditure among youth [21]. These accelerometers are small $(4.6 \times 3.3 \times 1.5 \mathrm{~cm})$, light weight $(19 \mathrm{~g})$ triaxial accelerometers that are considered reliable (intra-class correlation $=0.97$ ) for most common types of human locomotion [22] and accurately measure physical activity compared to oxygen consumption [23]. The participants wore the accelerometer on their right hip for five consecutive weekdays with a 2-second epoch $(30 \mathrm{~Hz})$. The selected daily time range for PA was analyzed for afterschool hours from 16:00-22:00. The minimum wearing time was $4 \mathrm{~h}$ afterschool for this study. We used Puyau, Adolph, Vohra, and Butte (2002)'s cutoff criteria to calculate sedentary $(<800$ count per minute $(\mathrm{CPM}))$, light $(<3200 \mathrm{CPM})$, moderate $(<8200 \mathrm{CPM})$, vigorous $(\geq 8200 \mathrm{CPM})$ time, respectively [24]. These cutoffs were used because they were shown to be sensitive to the 
pediatric population [25]. Accelerometer data were processed through ActiLife 6 software (ActiGraph, Pensacola, FL, USA) and were included in the analysis if the daily minimum wearing time was met. The aggregated daily average was used to describe adolescent weekday afterschool physical activity.

\subsection{Procedure}

This study used an observational research design to examine the association between health-related fitness performances and accelerometer-measured afterschool physical activity during weekdays. This study was conducted in a naturalistic environment, with no intervention involved. This study was conducted in a suburban school district located in the Eastern United States, where the schools had ample equipment for physical education and the majority of the residents were middle class. The schools typically have two to three physical education classes weekly depending on the schedule.

After the university institutional review board and school district research office approved the study protocols (approval \#: 12-106), the researchers distributed parental consent and student assent forms for participant recruitment. Upon receiving the consent/assent forms, the data collection began with fitness tests. Following the standard testing protocols specified in FITNESSGRAM [8], the researchers conducted the tests with physical educator's presence in school. The researchers brought accelerometers to school, provided instruction, and allowed students to use them for two days to mitigate reactivity. Then the researchers distributed the programmed accelerometers for data collection. Once the wearing time completed, the researchers collected the accelerometers, downloaded the accelerometry data, and processed them for data analyses.

\subsection{Data Analysis}

We ran descriptive statistics on health-related fitness components and physical activity variables. Then, we conducted Pearson product-moment correlations between health-related fitness performances and physical activity variables. Finally, a multiple regression analysis with student afterschool MVPA as the dependent variable and health-related fitness components as independent variables was used to determine the predictive value of health-related fitness components for student afterschool MVPA. Statistical significance level was held at $\alpha=0.05$ during the analyses, which was conducted using SPSS (Ver. 25, IBM; Armonk, NY, USA).

\section{Results}

As shown in Table 1, while on average the adolescents' health-related fitness test results were within the health-related fitness zone, their afterschool hours were mostly sedentary $(274.27 \pm 66.89 \mathrm{~min})$ and doing light physical activity $(73.68 \pm 51.66 \mathrm{~min})$, and only $11.35 \pm 16.92 \mathrm{~min}$ of MVPA, accumulating approximately $2058.52 \pm 1690.56$ steps each day afterschool. As displayed in Table 2, PACER performance (cardiorespiratory endurance) was the only fitness component significantly correlated with afterschool MVPA $(r=0.39)$, energy expenditure $(r=0.40)$, step count $(r=0.31)$, sedentary time $(r=-0.23)$, and BMI $(r=-0.29)$. The other health-related fitness test components, including BMI, did not significantly correlate with physical activity variables ( $p s>0.05,|r|<0.20)$.

As shown in Table 3, the multiple regression analysis results suggested that a significant amount of the variance in adolescent afterschool MVPA was explained by the model $\left(F_{7,89}=3.17, p<0.05\right)$. Overall, the model explained approximately $22.8 \%$ of the variance in the participants' MVPA, with gender, BMI, PACER, sit-and-reach, trunk lift, curl-up, and push-up as predictors. According to Cohen [26], this result $\left(f^{2}=0.29\right)$ indicates a medium effect size $\left(f^{2}>0.15\right)$ [26]. PACER test performance was the only variable that emerged as a significant positive predictor for adolescent afterschool MVPA $(\beta=0.47, p<0.05)$. 
Table 1. Descriptive results of the variables.

\begin{tabular}{|c|c|c|c|c|}
\hline Variables & $\mathbf{M}$ & SD & Min. & Max. \\
\hline Sedentary $\left(\mathrm{min} \cdot \mathrm{d}^{-1}\right)$ & 274.27 & 66.89 & 148.00 & 457.00 \\
\hline Light PA $\left(\min \cdot \mathrm{d}^{-1}\right)$ & 73.68 & 51.66 & 10.00 & 200.00 \\
\hline PA count (cpm) & $96,049.57$ & $106,227.81$ & 157.00 & $634,592.00$ \\
\hline Step count $\left(n \cdot d^{-1}\right)$ & 2058.52 & 1690.56 & 208.00 & 8299.00 \\
\hline $\mathrm{EE}(\mathrm{kcal})$ & 84.93 & 77.95 & 20.84 & 507.13 \\
\hline $\operatorname{MVPA}\left(\min \cdot \mathrm{d}^{-1}\right)$ & 11.35 & 16.92 & 0.00 & 98.00 \\
\hline $\mathrm{BMI}\left(\mathrm{kg} \cdot \mathrm{m}^{-2}\right)$ & 19.35 & 2.56 & 14.06 & 25.05 \\
\hline PACER (lap) & 37.18 & 14.21 & 16.00 & 81.00 \\
\hline Sit-and-reach $(\mathrm{cm})$ & 11.03 & 2.73 & 5.00 & 18.00 \\
\hline Trunk lift $(\mathrm{cm})$ & 10.46 & 1.60 & 6.00 & 12.00 \\
\hline Curl-up (n) & 47.83 & 21.89 & 11.00 & 80.00 \\
\hline Push-up (n) & 15.19 & 7.32 & 1.00 & 33.00 \\
\hline
\end{tabular}

Note: PA = physical activity; CPM = Count Per Minute; $\mathrm{M}=$ mean; SD = standard deviation; EE = energy expenditure; MVPA = moderate-to-vigorous physical activity; $\mathrm{BMI}=$ body mass index; PACER = Progressive Aerobic Cardiovascular Endurance Run.

Table 2. The Pearson product-moment correlation coefficients between variables.

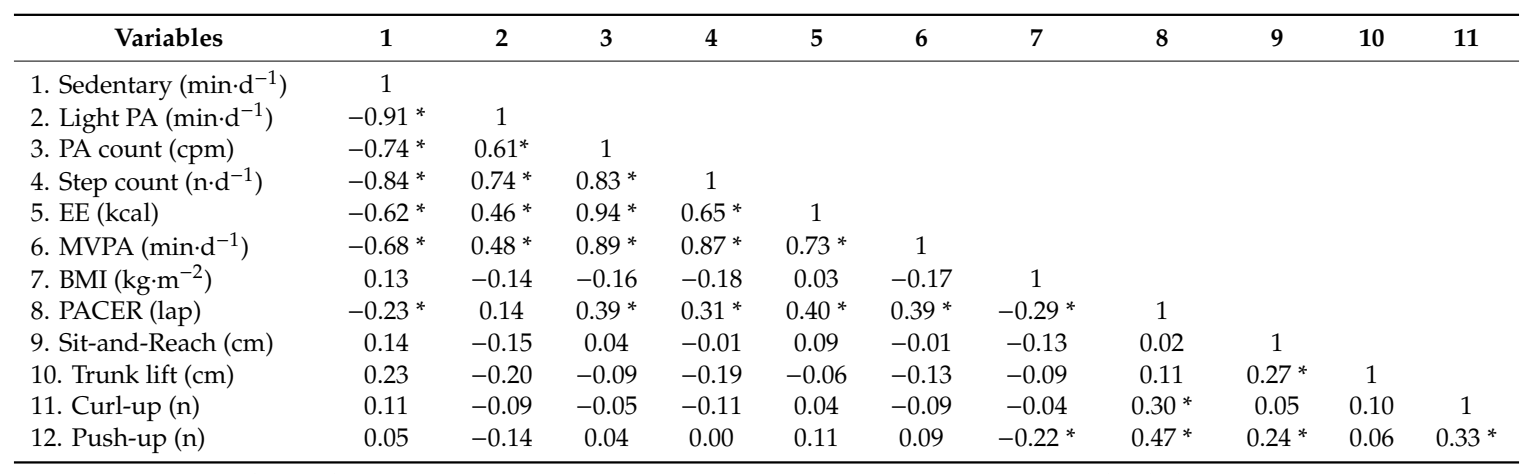

Note: PA = physical activity; $\mathrm{CPM}=$ Count Per Minute; $\mathrm{M}=$ mean; $\mathrm{SD}=$ standard deviation; $\mathrm{EE}=$ energy expenditure; MVPA = moderate-to-vigorous physical activity; $\mathrm{BMI}=$ body mass index; PACER = Progressive Aerobic Cardiovascular Endurance Run, ${ }^{*} p<0.05$.

Table 3. Multiple regression results predicting afterschool moderate-to-vigorous physical activity.

\begin{tabular}{|c|c|c|c|c|c|}
\hline \multirow{2}{*}{ Predictors } & \multicolumn{5}{|c|}{$R^{2}=22.8 \%, F_{7,89}=3.17, p<0.05$} \\
\hline & B & $\mathrm{SE}_{\mathrm{B}}$ & $\beta$ & $t$ & $p$ \\
\hline Intercept & 21.54 & 12.43 & & 0.96 & 0.34 \\
\hline Gender & 0.97 & 0.58 & 0.03 & 0.19 & 0.85 \\
\hline $\mathrm{BMI}\left(\mathrm{kg} \cdot \mathrm{m}^{-2}\right)$ & -0.38 & 0.37 & -0.06 & -0.52 & 0.61 \\
\hline PACER (lap) & 0.53 & 0.15 & $0.47^{*}$ & 3.55 & 0.00 \\
\hline Sit-and-reach (cm) & 0.52 & 0.19 & 0.09 & 0.57 & 0.57 \\
\hline Trunk lift (cm) & -1.88 & 1.18 & -0.18 & -1.59 & 0.12 \\
\hline Curl-up (n) & -0.16 & 0.10 & -0.21 & -1.68 & 0.10 \\
\hline Push-up (n) & -0.15 & 0.15 & -0.06 & -0.42 & 0.67 \\
\hline
\end{tabular}

Note: $\overline{\mathrm{BMI}}=$ body mass index; PACER $=$ Progressive Aerobic Cardiovascular Endurance Run, ${ }^{*} p<0.05$.

\section{Discussion}

The GAO reported that the instructional time for physical education and the percentage of schools offering physical education at least 3 days a week in all K-12 grades has declined due to increased pressure for academic assessments [5]. However, as noted by the GAO report, students' opportunities to participate in afterschool sports had increased during the same period [5]. The GAO report suggested that increased sport programs and other physical activity opportunities after school should play an important role in improving students' weekly physical activity and the benefits associated with physical 
activity participation [5]. The U.S. Department of Health and Human Services recommends a minimum of $60 \mathrm{~min}$ of MVPA per day for adolescents [3]. Clearly, most adolescents in this study did not meet targeted amounts of physical activity relying on afterschool physical activity alone. These results provide further justification that physical education and other school-based physical activity must play an important role in supplementing and promoting student physical activity [27].

A unique aspect of our investigation was the use of cardiorespiratory endurance as a predictor of afterschool physical activity. Much of the previous literature examining the associations between these variables used physical activity as a predictor of cardiorespiratory endurance [12], which is not surprising given the large body of evidence showing that increasing physical activity, particularly MVPA, leads to improvements in cardiorespiratory fitness [28]. However, cardiorespiratory fitness levels can prospectively predict future risk of a number of important health-related variables, including physical activity engagement $[19,20]$. Cardiorespiratory fitness may influence a child's likelihood of participating in physical activity in both direct and indirect ways. A physically fit child may enjoy physical activity more, especially vigorous activity, as compared to a child with poor cardiorespiratory fitness. More indirectly, cardiorespiratory fitness may act as a surrogate marker for other factors that influence physical activity participation, such as neuromotor development and coordination. Regardless of the mechanism, future studies should consider examining cardiorespiratory fitness as a predictor of different types of physical activity and sedentary behaviors among children.

Our results are not completely aligned with those from Morrow and Freedson [12], but they are consistent with other more recent findings $[11,16]$ in that cardiorespiratory endurance is moderately correlated with afterschool MVPA. The advancement in the measurement and evaluation [17] could be a major factor influencing the differences. Over 20 years ago, many of the currently available objective PA measures were not available. Indeed, Morrow and Freedson's review that found only a weak correlation (0.16-0.17) between physical activity and aerobic fitness primarily examined questionnaire-based studies, with a select few utilizing actometers or other early, simple versions of activity monitors. Nevertheless, as evidenced in our study and others [11-14,16], it is consistent that cardiorespiratory endurance is not significantly associated with light physical activity.

In addition to cardiorespiratory endurance, we included other health-related measures including BMI in our analyses. Interestingly, BMI and other measures were not significantly correlated with adolescent afterschool MVPA, even though BMI is significantly negatively correlated with cardiorespiratory endurance. Previous studies have pointed out mixed findings on the relationship between BMI, cardiorespiratory endurance, and physical activity [13-15]. In short, however, our regression analysis results suggest that health-related fitness measures and gender, as evidenced by the model $R^{2}=22.8 \%$, have a positive moderate association with afterschool MVPA. Clearly, there are other factors that could contribute to explaining the remaining variance in afterschool MVPA.

Considering the fact that for many adolescents in middle school, their daytime is tightly scheduled, what is unique in our study is that we measured their daily physical activity during afterschool hours when they may have the chance to voluntarily participate in activities. This study suggests that promoting health-related fitness (particularly cardiorespiratory endurance) in physical education is relevant to improving student afterschool physical activity, which is consistent with a standard expectation of physical education [14]. Physical educators should continue to explore ways to engage students in fitness education and fitness testing process $[6,29,30]$. Future studies should continue to look at the relevance and contribution of the other health-related fitness components in promoting MVPA afterschool.

Limitations of this preliminary study include the relatively small sample size may prevent generalization to a wider population. The sample is also limited to middle class communities and 11-13 years old, which may reduce generalizability for children living in lower income households or other age group. Additionally, we did not measure contextual/behavioral variables such as what types of activities the participants partook, if they participated in activities individually or as a group, or the location of such activities. 


\section{Conclusions}

In summary, this study demonstrated the association between cardiovascular endurance test results and afterschool physical activity, with a moderate effect size in the explained variance of afterschool MVPA. That is, fit students do tend to exercise more after school, particularly those who have higher aerobic fitness. Additionally, this study showed that the majority of time spent by adolescents during afterschool hours was associated with sedentary behaviors or light physical activity, with few activities eliciting MVPA values. This is of concern, because recent declines in physical education time in schools because of high focus on academic testing allow for little school-based time for youth to be physically active. In an effort to reallocate when adolescents would gain important physical activity minutes, organizations like the GAO (2012) have targeted afterschool hours [5]. However, these results show that this may not be the case. These findings can inform professionals of the need to provide structured opportunities for physical activity during afterschool hours to ensure that this time contributes to the recommended $60 \mathrm{~min}$ of daily MVPA for children.

Author Contributions: Conceptualization, D.Y. and X.Z.; Data curation, D.Y. and X.Z.; Formal analysis, X.Z.; Funding acquisition, X.W.; Investigation, J.A.H.; Project administration, X.W.; Writing-original draft, X.Z.; Writing-review \& editing, D.Y., X.Z., J.A.H. and P.B.W.

Funding: This research was in part supported by National Social Science Foundation of China (Grant No. 18ATY004).

Acknowledgments: The researchers thank the children for their participation in this study. The first author was a visiting scholar at Old Dominion University when the data were collected.

Conflicts of Interest: The authors declare no conflict of interest. The funders had no role in the design of the study; in the collection, analyses, or interpretation of data; in the writing of the manuscript, or in the decision to publish the results.

\section{References}

1. Bailey, D.; Boddy, L.; Savory, L.; Denton, S.; Kerr, C. Associations between cardiorespiratory fitness, physical activity and clustered cardiometabolic risk in children and adolescents: The HAPPY study. Eur. J. Pediatr. 2012, 171, 1317-1323. [CrossRef] [PubMed]

2. Zhu, X.; Haegele, J.A.; Healy, S. Movement and mental health: Behavior correlates of anxiety and depression among children of 6-17 years old in the U.S. Ment. Health Phys. Act. 2019, 16, 60-65. [CrossRef]

3. U.S. Department of Health and Human Services. Physical Activity Guidelines for Americans, 2nd ed.; U.S. Department of Health and Human Services: Washington, DC, USA, 2018.

4. America Society of Health and Physical Educators (SHAPE). National Standards E Grade-Level Outcomes for K-12 Physical Education; SHAPE: Reston, VA, USA, 2014.

5. Government Accountability Office. School-Based Physical Education and Sport Programs; Government Accountability Office: Washington, DC, USA, 2012.

6. Silverman, S.; Keating, X.; Philips, S. A lasting impression: A pedagogical perspective on youth fitness testing. Meas. Phys. Educ. Exerc. Sci. 2008, 12, 146-166. [CrossRef]

7. Morrow, J.R. 1958-2008: 50 years of youth fitness tests in the United States. Res. Q. Exerc. Sport 2009, 80, 1-11. [CrossRef] [PubMed]

8. Plowman, S.A.; Meredith, M.D. (Eds.) Fitnessgram/Activitygram Reference Guide; The Cooper Institute: Dallas, TX, USA, 2013.

9. Armstrong, N. Top 10 research questions related to youth aerobic fitness. Res. Q. Exerc. Sport 2017, 88, 130-148. [CrossRef] [PubMed]

10. Cale, L.; Harris, J. Fitness testing in physical education-A misdirected effort in promoting healthy lifestyles and physical activity? Phys. Educ. Sport Pedagog. 2009, 14, 89-108. [CrossRef]

11. Aires, L.; Silva, P.; Silva, G.; Santos, M.P.; Ribeiro, J.C.; Mota, J. Intensity of physical activity, cardiorespiratory fitness, and body mass index in youth. J. Phys. Act. Health 2010, 7, 54-59. [CrossRef]

12. Morrow, J.R.; Freedson, P.S. Relationship between habitual physical activity and aerobic fitness in adolescents. Pediatr. Exerc. Sci. 1994, 6, 315-329. [CrossRef] 
13. Gutin, B.; Yin, Z.; Humphries, M.C.; Barbeau, P. Relations of moderate and vigorous physical activity to fitness and fatness in adolescents. Am. J. Clin. Nutr. 2005, 81, 746-750. [CrossRef]

14. Lohman, T.G.; Ring, K.; Pfeiffer, K.; Camhi, S.; Arredondo, E.; Pratt, C.; Pate, R.; Webber, L.S. Relationships among fitness, body composition, and physical activity. Med. Sci. Sports Exerc. 2008, 40, 1163-1170. [CrossRef]

15. Rauner, A.; Mess, F.; Woll, A. The relationship between physical activity, physical fitness and overweight in adolescents: A systematic review of studies published in or after 2000. BMC Pediatr. 2013, 13, 19. [CrossRef] [PubMed]

16. Ross, R.; Mcguire, K.A. Incidental physical activity is positively associated with cardiorespiratory fitness. Med. Sci. Sports Exerc. 2011, 43, 2189-2194. [CrossRef] [PubMed]

17. Mood, D.P.; Jackson, A.W.; Morrow, J.R. Measurement of physical fitness and physical activity: Fifty years of change. Meas. Phys. Educ. Exerc. Sci. 2007, 11, 217-227. [CrossRef]

18. Burn, N.L.; Weston, M.; Maguire, N.; Atkinson, G.; Weston, K.L. Effects of workplace-based physical activity interventions on cardiorespiratory fitness: A systematic review and meta-analysis of controlled trials. Sports Med. 2019, 49, 1255-1274. [CrossRef] [PubMed]

19. Dennison, B.A.; Straus, J.H.; Mellits, E.D.; Charney, E. Childhood physical fitness tests: Predictor of adult physical activity levels? Pediatrics 1988, 82, 324-330. [PubMed]

20. Glenmark, B.; Hedberg, G.; Jansson, E. Prediction of physical activity level in adulthood by physical characteristics, physical performance and physical activity in adolescence: An 11-year follow-up study. Eur. J. Appl. Physiol. 1994, 69, 530-538. [CrossRef]

21. Sasaki, J.; John, D.; Freedson, P. Validation and comparison of ActiGraph activity Monitors. J. Sci. Med. Sport 2011, 14, 411-416. [CrossRef] [PubMed]

22. Santos-Lozano, A.; Marín, P.J.; Torres-Luque, G.; Ruiz, J.R.; Lucía, A.; Garatachea, N. Technical variability of the GT3X accelerometer. Med. Eng. Phys. 2012, 34, 787-790. [CrossRef] [PubMed]

23. Kelly, L.A.; McMillan, D.G.; Anderson, A.; Fippinger, M.; Fillerup, G.; Rider, J. Validity of actigraphs uniaxial and triaxial accelerometers for assessment of physical activity in adults in laboratory conditions. BMC Med. Phys. 2013, 13, 5. [CrossRef]

24. Puyau, M.R.; Adolph, A.L.; Vohra, F.A.; Butte, N.F. Validation and calibration of physical activity monitors in children. Obes. Res. 2002, 10, 150-157. [CrossRef]

25. Gába, A.; Dygrýn, J.; Mitáš, J.; Jakubec, L.; Frömel, K. Effect of accelerometer cut-off points on the recommended level of physical activity for obesity prevention in children. PLoS ONE 2016, 11, e0164282. [CrossRef] [PubMed]

26. Cohen, J. Statistical Power Analysis for the Behavioral Sciences, 2nd ed.; Lawrence Earlbaum Associates: Hillsdale, NJ, USA, 1988.

27. Pate, R.R.; Davis, M.G.; Robinson, T.N.; Stone, E.J.; McKenzie, T.L.; Young, J.C.; American Heart Association Council on Nutrition, Physical Activity, and Metabolism (Physical Activity Committee); Council on Cardiovascular Disease in the Young; Council on Cardiovascular Nursing. Promoting physical activity in children and youth: A leadership role for schools: A scientific statement from the American Heart Association Council on Nutrition, Physical Activity, and Metabolism (Physical Activity Committee) in collaboration with the Councils on Cardiovascular Disease in the Young and Cardiovascular Nursing. Circulation 2006, 114, 1214-1224. [PubMed]

28. Haskell, W.L.; Lee, I.M.; Pate, R.R.; Powell, K.E.; Blair, S.N.; Franklin, B.A.; Macera, C.A.; Heath, G.W.; Thompson, P.D.; Bauman, A. Physical activity and public health: Updated recommendation for adults from the American College of Sports Medicine and the American Heart Association. Circulation 2007, 116, 1081-1093. [CrossRef] [PubMed]

29. Zhu, X. Situational interest and physical activity in fitness testing: A need for pedagogical engineering. Int. J. Sport Exerc. Psychol. 2014, 12, 76-89. [CrossRef]

30. Zhu, X.; Chen, S. Effects of cognitive demand on situational interest and running task performances. Educ. Psychol. 2017, 37, 907-920. [CrossRef]

(C) 2019 by the authors. Licensee MDPI, Basel, Switzerland. This article is an open access article distributed under the terms and conditions of the Creative Commons Attribution (CC BY) license (http://creativecommons.org/licenses/by/4.0/). 